\title{
Una lunga strada di parole
}

Una memoria fortunosamente ritrovata è il cuore della narrazione. Attraverso le lettere dal fronte di Severino Belenghi, un giovane soldato dichiarato disperso in Russia nel dicembre 1942, è stata ricostruita e raccontata la sua storia, lunga sedici mesi, nel contesto tragico della campagna di Russia e in quello personale e privatissimo di un dialogo ininterrotto con i familiari. L'autrice, nipote di Severino Belenghi, ha tradotto in performance teatrale questa narrazione e l'ha presentata come prova conclusiva della terza edizione del Master di Comunicazione storica dell'Università di Bologna.

A fortunately found memory is the core of the narration. Severino Belenghi was a young soldier declared missing in Russia in December 1942. Through his letters from the front, his sixteen-month-long story was reconstructed in the tragic context of the Russian campaign and in his personal and very private one, made of an uninterrupted dialogue with his family. The author, Severino Belenghi's niece, has turned this narration into a theatrical performance and presented it as her conclusive work in the third edition of the Master di Comunicazione storica of the University of Bologna.

(Contributo video disponibile online: 䁌 https://www.youtube.com/ watch?v=acwA1Qo6zuM).

1. Una memoria ritrovata

Al centro del lavoro che ho presentato come prova conclusiva della terza edizione del Master di Comunicazione storica dell'Università di Bologna, e il cui titolo è lo stesso di questo contributo, ci sono le lettere che un soldato inviò, con regolarità quasi cronometrica, dal fronte russo alla propria numerosa famiglia. 
Tra le molte fonti che possono essere utilizzate per tentare di capire l'esperienza tanto assurda quanto traumatica della guerra in generale, e di quella combattuta in Russia in particolare, le lettere, fonte creata nel divenire dell'esperienza belli$\mathrm{ca}$, sono quelle più dirette: ci comunicano emozioni, paure, speranze, bisogni ed anche i sottintesi o i non detti sono carichi di significato. Se è innegabile, quindi, l'importanza oggettiva di questa fonte storica, c'è, tuttavia, nelle lettere che ho esaminato, trascritto, studiato, sulle quali ho riflettuto una valenza del tutto speciale che riguarda sia il loro mittente che i destinatari, perché si tratta della mia famiglia.

La storia che quelle lettere raccontano è quella di un giovane uomo mandato a combattere in Russia e mai più tornato a casa, "disperso in Russia" come annunciava il laconico quanto lapidario epilogo ufficiale alla sua brevissima vita, 28 anni appena. Quel giovane si chiamava Severino Belenghi, era nato nel 1914 in un paesino dal nome gentile, Soave, pochi chilometri da Mantova, ed era uno dei fratelli di mio padre.

L'idea di dare forma e visibilità alla storia di Severino credo mi sia sempre appartenuta, fin da quando, bambina, ne sentivo parlare da mio padre: i suoi racconti erano molto scarni, fatti soprattutto di omissioni, di autocensure difensive che allora non capivo ma che ho capito anni dopo quando ho cominciato ad ascoltare le testimonianze di partigiani e reduci dai campi di prigionia che venivano a scuola per parlare con i miei alunni. Senza scendere nel dettaglio dell'attendibilità storica, dell'oggettività scientifica delle loro testimonianze e dei loro ricordi ho comunque capito che tutti loro avevano in comune una cosa, un silenzio durato decenni che se da un lato impediva ai ricordi di fluire dall'altro proteggeva quelle persone dallo scontrarsi faccia a faccia con un dolore che non si poteva dire perché non esistevano parole per dirlo. La stessa cosa, probabilmente, aveva fatto mio padre, si era protetto dietro il silenzio.

A me non piaceva quel racconto scarno e, caparbiamente, cercavo di trovare un senso, una logica per quella storia, ma ogni tentativo si perdeva nel vuoto: dapprima l'impossibilità di avere qualsiasi tipo di notizia durante gli anni della "guerra fredda”, poi, con la dissoluzione dell'Unione Sovietica, ci furono delle persone, individui più o meno discutibili, che si fecero pubblicità facendo nascere nei parenti dei "dispersi" l'illusione di far loro avere quelle notizie che mancavano da oltre quarant'anni.

Malvolentieri mio padre raccontava, ed era il suo racconto più lungo, di quando era andato a Pescantina di Verona ad aspettare i convogli che attraverso la Polonia riportavano in patria i sopravvissuti alla campagna di Russia: qualche fotografia e molta speranza lo sostenevano mentre si aggirava tra quei fantasmi che scen- 
devano dalle tradotte, mentre chiedeva e si sentiva rispondere di aspettare l'altro treno, il terzo che era partito ma che non arrivò mai. Nelle richieste di notizie che cominciammo a spedire insieme, nelle quali io cercavo di dare un senso, o forse un ordine ai pochi frammenti che avevo a disposizione, si accennava anche ad una fantomatica trasmissione ultra clandestina da Radio Mosca del 6 gennaio 1943 nella quale Severino si sarebbe detto vivo, in buona salute ed in procinto di tornare a casa. Millantatori? Sciacalli? L'unica cosa certa è che Severino non tornò mai.

Sarebbero passati molti anni prima che io avessi notizia dell'esistenza di un gruppo di ricerca che si occupava di rintracciare i "dispersi" mantovani della campagna di Russia; le notizie che ricevetti da loro contrastavano con i miei racconti e mi dicevano, invece, una verità molto attendibile, quella della più che probabile morte di Severino, in combattimento, avvenuta nel dicembre 1942, in una località non ben definita. Una piccola mappa con le diciture in russo accompagnava la lettera e, per quanto ne sapevo, in quel foglio fotocopiato erano racchiuse la vita e la morte di Severino. Era ancora troppo poco ma, almeno, era un piccolo passo avanti (che tuttavia non potei condividere con mio padre: lui se n'era già andato tre anni prima).

La svolta avvenne qualche anno fa quando, durante una conversazione con uno dei miei cugini, venni in possesso di un piccolo patrimonio di 172 lettere e biglietti, a volte anche di una sola riga, scritti da Severino durante tutto il periodo della sua permanenza in Russia, sedici mesi ininterrotti durante i quali aveva cercato in ogni modo di non perdere il contatto con la sua famiglia per non sentirsi solo, per non impazzire, per dare e darsi il coraggio necessario a mettere i giorni in fila, uno dopo l'altro. Finalmente Severino non era più solo un nome a cui la fotografia che mio padre teneva sul comodino accanto al letto dava un volto, non era più solo un soldato in divisa ma era anche una voce che mi raccontava una storia, la sua storia ed io non potevo fare altro che ascoltarla e mettermi in cammino sulla lunga strada di parole che Severino aveva tracciato.

\section{La storia}

La storia che emerge dalle lettere comincia nell'estate del 1941: in giugno Hitler aveva attaccato l'Unione Sovietica e Mussolini aveva prontamente inviato a sostegno dell'alleato tedesco un'unità chiamata Csir composta da circa 60.000 uomini distribuiti in tre divisioni: "Celere", dotata di mezzi veloci, autocarri e motociclette; "Pasubio" e "Torino", definite con imbarazzate entusiasmo autotrasportabili ma praticamente appiedate. Equipaggiate con armamenti antiquati 
e vestiario inadatto al clima russo, nell'estate 1941 dalle stazioni di Cremona, Verona e Roma cominciarono a muoversi le prime tradotte destinazione Russia [Bedeschi 1963; Carrel 2000; Scotoni 2010 e 2012].

Tra i soldati della "Pasubio", inquadrato nei ranghi dell' $80^{\circ}$ Fanteria, in partenza da Verona, c'era anche Severino. Per lui non si trattava di un battesimo del fuoco: era stato richiamato in servizio in marzo, mandato in Jugoslavia, da lì richiamato in fretta e furia per andare a combattere in Russia. Il viaggio fu lungo attraverso mezza Europa fino al fiume Bug che segnava il nuovo confine dell'Unione Sovietica.

Le lettere che Severino mandava in quel periodo raccontano il cauto ottimismo di chi, pur andando incontro all'ignoto, non può e non vuole allarmare la famiglia, e allora meglio raccontare del paesaggio, dei chilometri fatti, del viaggio. Poi, nello scorrere dei mesi, la vita militare si fa sempre più dura, la guerra continua $\mathrm{e}$ gli umori cambiano. Dalla scrittura di Severino emerge sempre più evidente una dicotomia: da un lato il senso di colpa nei confronti della famiglia, della madre in particolare, per la difficoltà di inviare notizie, accompagnata dalla necessità quasi fisica di sapere la madre serena, "allegra", come raccomanda a lei e soprattutto ai fratelli; e dall'altro l'incapacità di continuare sempre a fingere che tutto vada bene, che non ci siano pericoli, che il freddo, la fame, i pidocchi, le scarpe rotte, il rischio concreto e quotidiano di morire altro non siano che un'invenzione della propaganda nemica. E allora, nonostante la censura, si leggono anche parole di cedimento [Cortesi 2008; Bonasea 1999; Pollorini 1971; Bellomo 1975; Revelli 1966 e 1971; Rizzi 1984].

La situazione peggiorò nel 1942, con la creazione dell'Armir, quando, oltre all'aumento degli uomini al fronte e delle difficoltà conseguenti, cominciarono anche a circolare insistenti voci di rimpatrio per i soldati che da più tempo si trovavano al fronte. Severino ci sperò con tutto se stesso al punto che, quando il rimpatrio non avvenne, pensò addirittura di sposarsi pur di poter tornare a casa. Ogni cosa sembrava pronta, i documenti, i passi burocratici ma le carte si persero e tutti i suoi progetti matrimoniali sfumarono anche per la testarda opposizione materna a un matrimonio per procura al quale, forse, Severino aveva anche pensato per poter accelerare i tempi e rientrare nel gruppo degli "sposati" che certamente sarebbero tornati a casa, ma di fronte alla volontà materna non volle, o forse non poté, nemmeno ammettere di aver considerato la prospettiva.

La sua ultima lettera dal fronte porta la data del 30 novembre 1942: Severino aveva compiuto 28 anni da appena un mese. Stava male, soffriva di dolori reumatici, aveva la febbre alta e le sue parole, nonostante le consuete rassicurazioni, sembravano quelle di un vecchio in attesa di un evento che mettesse fine, una volta 
per tutte, alla «maledetta Russia». La fine non tarderà a raggiungerlo, poco prima di Natale, nell'abbraccio mortale della neve e del ghiaccio.

\section{Visibilità}

Dopo aver ricostruito la storia di Severino attraverso la lettura delle sue lettere mi si poneva il problema di come darle forma e visibilità. Il mezzo più efficace e più vicino alle mie corde mi è sembrato quello di costruire una performance teatrale che desse ampio spazio alle parole di Severino ma che le contestualizzasse storicamente nel tempo e nei luoghi. Devo confessare che per l'idea di fondo sono in debito con il romanzo di Elsa Morante, La Storia [Morante 1974], dal quale ho mutuato l'impianto narrativo della rappresentazione organizzato su due livelli di lettura: una piccola storia raccontata all'interno della grande Storia che c'è, è ineluttabilmente presente, eppure quasi si ritira e accetta un ruolo non di protagonista ma di comprimaria.

Parte della narrazione, quella relativa al contesto storico, l'ho riservata a me mentre per la voce delle lettere mi sono affidata ad una voce maschile, nella circostanza più adatta della mia. Ho scelto la forma della reading perché non ho voluto che nulla, movimento o scena, togliesse spessore al contenuto del racconto e distogliesse l'attenzione da esso. Inoltre ho voluto che l'alternanza delle due voci, quella narrante e quella recitante, fosse commentata da un coro di voci maschili che eseguissero dal vivo canzoni ispirate al contesto storico o da esso provenienti. Scegliere un commento musicale di tipo corale mi è sembrata la soluzione più appropriata per rendere viva, anche attraverso questo strumento, la coralità di un'esperienza fatta di tante singolarità da essa accomunate.

Infine ho presentato lo spettacolo il 31 ottobre scorso, a Bologna, nella bellissima e raccolta Sala del tribunale del Circolo ufficiali dell'esercito. La scelta del giorno non è stata casuale: il 26 ottobre 2014, infatti, Severino avrebbe compiuto 100 anni, e mi è sembrato giusto ricordarne la memoria anche così, mentre narravo ai presenti la sua storia che, in fondo, è un tassello della mia.

\section{Bibliografia}

Bedeschi G. 1963, Centomila gavette di ghiaccio, Milano: Mursia

Bellomo B. 1975, Lettere censurate, Milano: Longanesi

Bonasea G. 1999, Paesaggi al confine tra linguaggi e immagini sociali, "Storia e problemi contemporanei”, 24 
Carrel P. 2000, La campagna di Russia. 1941-1944, Milano: BUR

Cortesi E. 2008, Reti dentro la guerra. Corrispondenza postale e strategie di sopravvivenza (1940-1945), Roma: Carocci

Morante E. 1974, La Storia, Torino: Einaudi

Revelli N. 1966, La strada del davai, Torino: Einaudi

Revelli N. 1971, L'ultimo fronte. Lettere di soldati caduti o dispersi nella seconda guerra mondiale, Torino: Einaudi

Rizzi L. 1984, Lo sguardo del potere, Milano: Rizzoli

Scotoni G. 2010, Ricostruzione della disfatta italiana in Russia, Trento: Panorama

Scotoni G. 2012, L’Armata Rossa e la disfatta italiana (1942-1943), Trento: Panorama 